\title{
A novel hybrid multiple access scheme in downlink for 4G wireless communications
}

\author{
Poonam Singh ${ }^{1}$, Saswat Chakrabarti ${ }^{2}$ \\ ${ }^{1}$ Electronics \& Comm. Engg. Dept., National Institute of Technology, Rourkela, India \\ ${ }^{2}$ GSSST, Indian Institute of Technology, Kharagpur, India \\ Email address: \\ psingh@nitrkl.ac.in(P. Singh), saswat@ece.iitkgp.ernet.in(S. Chakrabarti)
}

\section{To cite this article:}

Poonam Singh, Saswat Chakrabarti. A Novel Hybrid Multiple Access Scheme in Downlink for 4G Wireless Communications. International Journal of Wireless Communications and Mobile Computing. Vol. 1, No. 4, 2013, pp. 103-112.

doi: $10.11648 /$ j.wcmc.20130104.14

\begin{abstract}
In this paper, we present a hybrid multiple access scheme to increase the data rate and to provide more flexibility for $4 \mathrm{G}$ wireless communications. The proposed scheme utilizes a combination of the multiple access techniques TDMA, CDMA and OFDMA. It uses variable time slots, spreading factors and number of subcarriers to provide flexible data rates for different classes of users. The performance analysis of the proposed scheme is carried out for different modulation schemes used in downlink and expressions for multiple access interference (MAI) and bit error rate (BER) are derived. The parameters for this scheme are selected depending on available bandwidth, required data rates, number of users, type of service provided etc. It is found that this scheme provides better scalability and can support more number of users.
\end{abstract}

Keywords: Multiple Access Scheme, FDMA, TDMA, CDMA, OFDMA

\section{Introduction}

An important goal of next generation wireless system is the convergence of multimedia services such as speech, audio, video, image and data. This implies that a future wireless terminal should be able to connect to different networks in order to support various services by guaranteeing high speed data. It is, therefore, very important to find a suitable transmission technique with high spectral efficiency and robustness to various distortions.

A variety of wireless, mobile Internet-access systems exist which provide broadband access to the Internet e.g. WLAN, WMAN etc. Orthogonal Frequency Division Multiple Access (OFDMA) is a multiple access scheme in which each user occupies different subcarriers. It offers dynamic user capacity and lower interference to adjacent cells [1] [2]. It has been adopted in WiMAX (IEEE 802.16e standard) [3] [4] and it is an attractive candidate for Beyond $3 \mathrm{G}$ systems due to its ability to limit intersymbol interference caused by multipath channels. The MC-CDMA and MC-DS-CDMA modulations combine the benefits of OFDM and CDMA to support high data rate transmission [5 - 9]. The hybrid TD-CDMA technique, which is selected as a standard for TDD mode of $3^{\text {rd }}$ Generation Partnership Project (3GPP) [10] [11], uses a combination of FDMA, TDMA and CDMA for multiple access and allows a flexible use of the limited transmission resources. Singlecarrier FDMA (SC-FDMA) is a hybrid modulation scheme that combines the low PAPR of single-carrier systems with the multipath resistance and flexible subcarrier frequency allocation offered by OFDM and has been proposed for the uplink of 3GPP Long Term Evolution (LTE) [12].

In this paper, we present a hybrid multiple access scheme to increase the data rate and to provide more flexibility for $4 \mathrm{G}$ wireless communications e.g. LTE, LTE-Advanced and IEEE $802.16 \mathrm{~m}$. The proposed scheme including its system model is described in Section II, which utilizes a combination of the multiple access techniques TDMA, CDMA and OFDMA. It uses variable time slots, spreading factors and number of subcarriers to provide flexible data rates for different classes of users. The performance analysis of the proposed scheme is carried out for different modulation schemes used in downlink in Sections III and expressions for multiple access interference (MAI) and bit error rate (BER) are derived. Some simulation results are given in Section IV. The parameters for this scheme are selected depending on available bandwidth, required data rates, number of users, type of service provided etc. Finally, 
the conclusions are given in Section V. It is found that this scheme provides better scalability and can support more number of users than what is mentioned in LTE proposals.

\section{A Hybrid Multiple Access Scheme (TD-MC-CDMA) in Synchronous Downlink}

Though the multicarrier methods like OFDMA, MCCDMA and MC-DS-CDMA can be used with TDM/TDMA for transmission, there is no explicit mention of TDMA application along with MC-CDMA in the existing literature. We would like to propose this combination for $4 \mathrm{G}$ applications to increase the scalability in the access. We are proposing variable spreading factors and number of time slots for different users depending on their data rates. This will provide a greater scalability, since the users with higher data rates will use lower spreading factor and will get more number of time slots. On the other hand, more number of users can be supported at low data rates with larger spreading factors. Analysis of OFDM and multicarrier spread spectrum modulation schemes have been done in many papers [13-20]. We have extended this analysis for the proposed TD-MC-CDMA system.

Fig. 1 shows the structure of transmitter for the proposed scheme for synchronous downlink. We consider a system with $\mathrm{K}$ users having different data rates. The data rate $\mathrm{R}_{\mathrm{k}}$ of user $\mathrm{k}(1 \leq \mathrm{k} \leq \mathrm{K})$ can be any one of the $\mathrm{N}_{\mathrm{d}}$ data rates supported by this scheme i.e. $R_{\min }<R_{k}<R_{\max }$. These $N_{d}$ data rates are integral multiples of the basic or minimum data rate $R_{\text {min }}$. The maximum data rate can be written as $\mathrm{R}_{\max }=\mathrm{N}_{\max } \mathrm{R}_{\min }$.

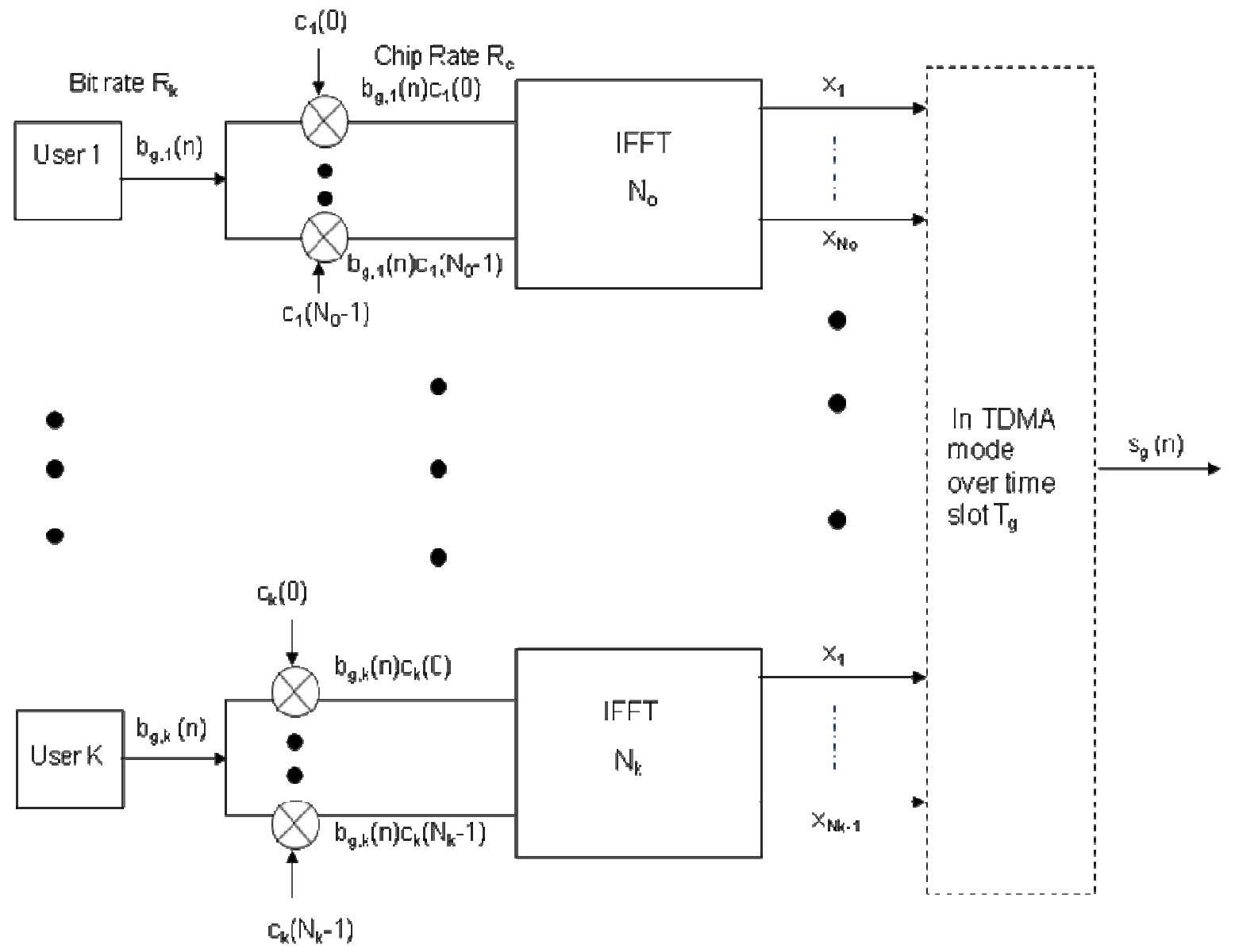

Fig.1. Transmission Scheme for users of group ' $g$ ' in the downlink

The complex valued data symbol $b_{g, k}$ of each user is multiplied with a spreading sequence $c_{g, k}$ of length $L$. The spreading factors are variable for different service classes, they are chosen depending on the data rates for each application. For lower data rates, a higher spreading factor and for higher data rates, a lower spreading factor is selected, so that the final chip rate $\mathrm{R}_{\mathrm{c}}$ for each user remains same. Thus, a large number of low data rate users or a small number of high data rate users can be accommodated in a given bandwidth. The spreading factor $\mathrm{Sf}$ for one application can be selected from a set of spreading factors $\mathrm{Sf}_{\min }$ to $\mathrm{Sf}_{\max },\left(\mathrm{Sf}_{\min } \leq \mathrm{Sf} \leq \mathrm{Sf}_{\max }\right)$. The complex valued data sequence for each user obtained after spreading is then modulated onto a number of subcarriers $\mathrm{N}_{\mathrm{k}}$.

The number of subcarriers is also variable and is equal to spreading factor for each user since each spread data is 
modulated on one subcarrier in this case. Thus each data symbol is spread over many subcarriers. As discussed in [8], the multicarrier CDMA schemes can be categorized into two groups: One (MC-CDMA) spreads the original data stream using a given spreading code and then modulates different subcarriers with each chip, i.e. spreading in the frequency domain. The other group (MC-DS-CDMA) spreads the serial to parallel converted data streams using a given spreading code and then modulates different carriers with each data stream i.e. spreading in the time domain similar to a conventional DS-CDMA scheme. In TD-MCCDMA scheme also, the chips of spread data symbol are transmitted in frequency direction over several parallel subchannels or in time direction over several multicarrier symbols. We propose TD-MC-CDMA with spreading in frequency direction for the downlink and spreading in time direction for the uplink in order to optimize both the spectral efficiency and mobile power consumption. The orthogonality among codes is maintained in the downlink so frequency domain spreading can be used to get frequency diversity. However, in the uplink, the transmitted signal of each user is affected by different channel impulse responses. So the orthogonality of signals is destroyed increasing MAI [20]. In time domain spreading, all the chips are transmitted on each of the subcarriers, so synchronization problems are reduced. In both the cases, variable time slots, spreading factors and variable number of subcarriers are used. In this paper, we analyze the multiple access scheme proposed for downlink only.

In one frame duration $\mathrm{T}_{\mathrm{F}}$, there is $\mathrm{N}_{\mathrm{s}}$ number of time slots. The number of time slots $\mathrm{N}_{\mathrm{k}}$ allocated to a user $\mathrm{k}$ depends on its data rate $\mathrm{R}_{\mathrm{k}}$. For example, the number of time slots allocated to a user with audio signal having a smaller data rate will be less as compared to time slots allocated to users having video or multimedia transmissions with higher data rates. A number of users with same data rates can be grouped together as a group $g$ and transmitted in one time slot. There can be maximum $G$ number of groups, which is equal to the number of time slots in a frame $\mathrm{N}_{\mathrm{g}}$. In each OFDM symbol, a guard time of $\mathrm{T}_{\mathrm{cp}}$ is usually inserted to reduce intersymbol interference. The OFDM symbol duration including a guard interval is $\mathrm{T}_{\text {sym }}=$ $\mathrm{T}+\mathrm{T}_{\mathrm{cp}}$, where $\mathrm{T}$ is the actual symbol duration. The maximum achievable data rate depends on the available channel bandwidth, number of time slots and the spreading factor.

The modulation scheme employed can be QPSK, 16QAM or 64-QAM depending on the data rate of users and channel conditions. For audio data which require a lower bandwidth and better BER at a given SNR, lower modulation like QPSK is used, whereas for video signals requiring a higher bandwidth and comparatively lower BER requirements, higher modulation schemes like 16QAM or 64-QAM is used. Adaptive modulation and coding can be used to improve the system efficiency.

Fig. 2 shows the receiver of user $\mathrm{k}$ in the TD-MC-CDMA system for downlink. In the receiver, after removing the guard interval and taking inverse OFDM the received sequence is equalized to get the transmitted data. For inverse OFDM, $\mathrm{N}_{\mathrm{k}}$ point FFT is taken and then frequency domain equalization is performed. After channel estimation, the output of each subcarrier is equalized and then coherently combined over the parallel subcarrier components using same code as used at the transmitter i.e. despreading of signals is performed to recover the transmitted binary data. The demodulation and decoding used at the receiver should be corresponding to modulation and coding used at the receiver. All these information are passed to receiver in the beginning of every frame in form of a preamble.

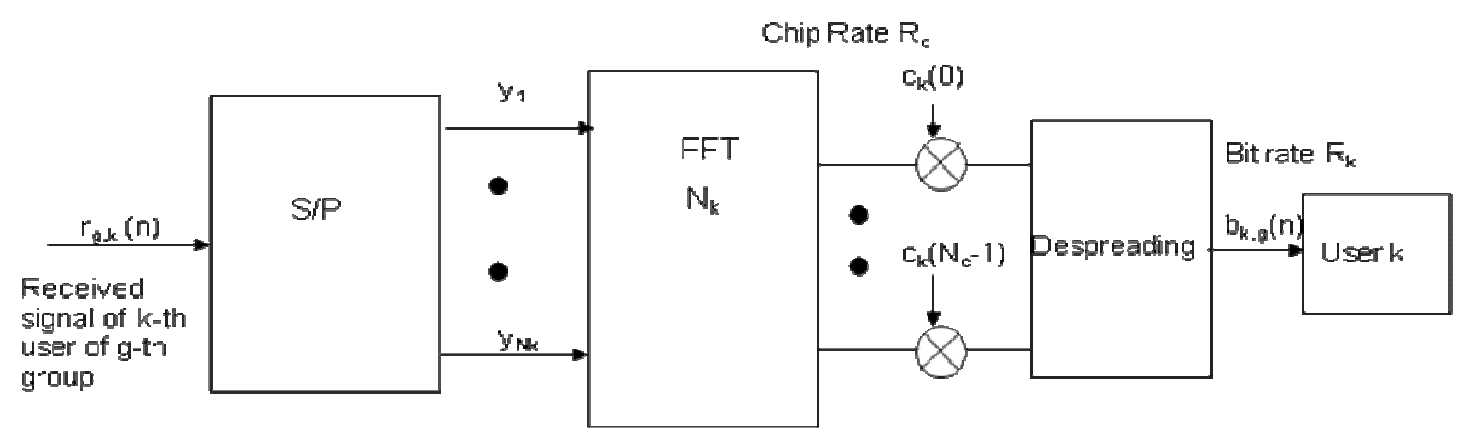

Fig.2. Receiver of user $k$ using TD-MC-CDMA for downlink

\subsection{Frame Structure for Downlink TD-MC-CDMA}

The frame structure for TD-MC-CDMA system is shown in Fig.3; a radio frame with duration of $\mathrm{T}_{\mathrm{F}}$ is subdivided into $\mathrm{N}_{\mathrm{s}}$ main time slots $\left(\mathrm{T} 1\right.$ to $\mathrm{TN}_{\mathrm{s}}$ ) of duration $\mathrm{T}_{\mathrm{g}}$ and a preamble $\mathrm{T}_{\text {pre }}$. The preamble consists of time slots for downlink synchronization (DL Sync) and uplink synchronization (UL Sync). The burst structure of the main time slots consists of data blocks, pilot signals and a guard period of duration $\mathrm{T}_{\mathrm{p}}$. The frame duration can be written as

$$
T_{F}=T_{p r e}+N_{s}\left(T_{g}+T_{p}\right)
$$

In the time domain the period of one OFDM block is $\mathrm{T}_{\text {sym }}$ including an effective block period of $\mathrm{T}$ and a cyclic prefix of $T_{c p}$. A time slot of $T_{g}$ is equivalent to $\mathrm{N}_{\mathrm{os}}$ OFDM symbols. $\mathrm{T}_{\mathrm{g}}$ can be written as

$$
T_{g}=N_{o s} T_{s y m}=N_{o s}\left(T+T_{c p}\right)
$$




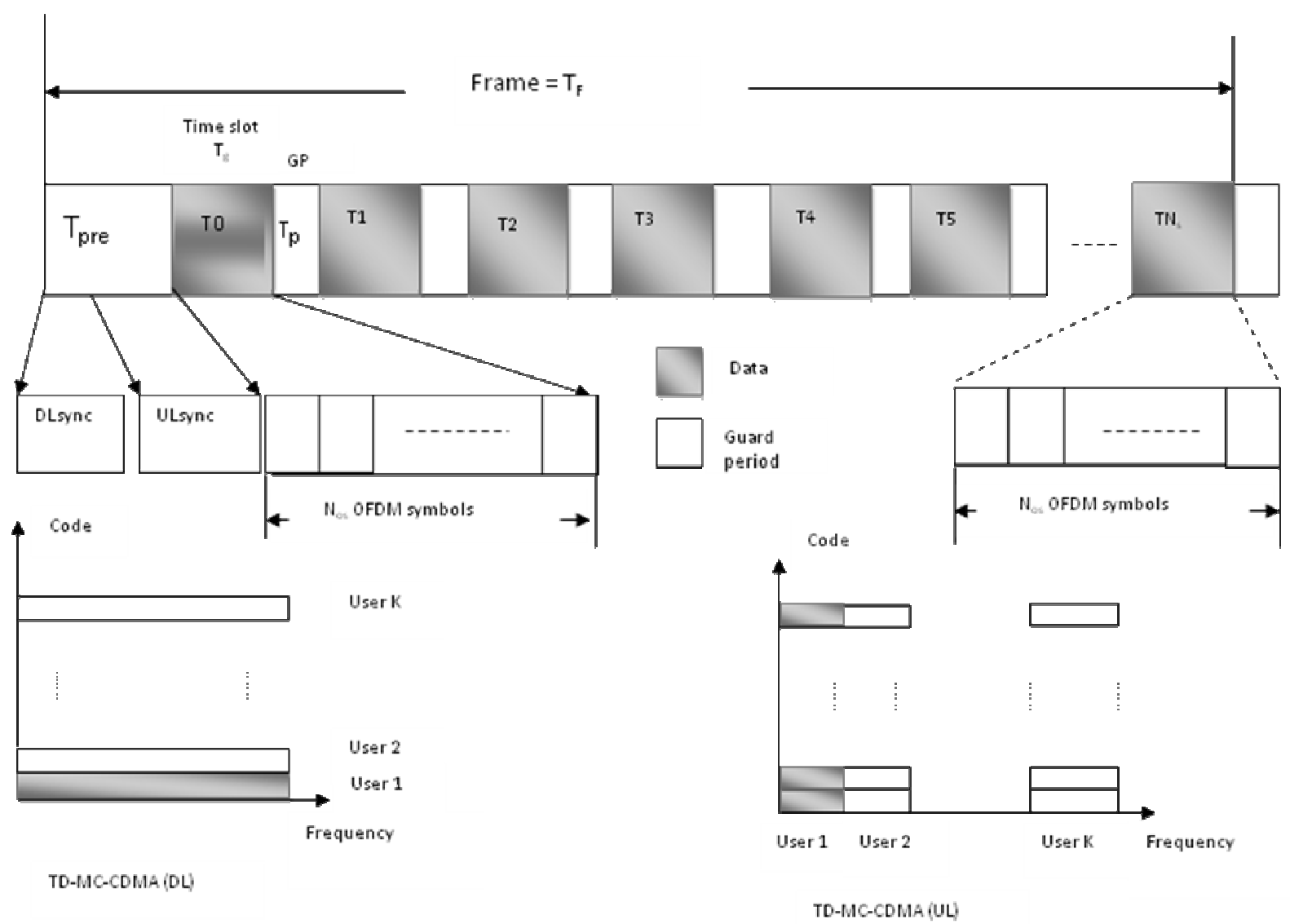

Fig.3. The frame structure for downlink of a TD-MC-CDMA system

\section{Performance Analysis}

We have taken the following assumptions for analysis:

a) There are $\mathrm{G}$ groups of $\mathrm{K}$ users each with data rates $\mathrm{R}_{\mathrm{k}}$ and binary spreading codes $\mathrm{c}_{\mathrm{k}}(\mathrm{t})$.

b) The transmitted binary data $b_{k}(k=1,2 \ldots K)$ for $K$ users are independent and identically distributed (i.i.d.) random variables.

c) Users can be assigned more than one time slot, but for simplicity we assume one time slot per frame for each user.

d) Signal power of the data bits $P_{s}$ is same for all users.

e) $w_{i}=w_{c}+2 \pi m / T_{b}$ is the $m$-th subcarrier frequency and $w_{c}$ is the radio frequency

f) $\theta_{\mathrm{k}}$ is the random carrier phase uniformly distributed over $[0,2 \pi]$.

g) Walsh codes are used for spreading since they are orthogonal.

h) The modulation scheme employed is QPSK.

i) The number of subcarriers $\mathrm{N}$ is equal to spreading factor for each user since each chip is modulated on one subcarrier in the downlink.

j) The statistics of all the Rayleigh fading channels are assumed to be identical for different users.
The input information data symbols $b_{k, g}(n)$ of k-th user of g-th group are first modulated using QPSK. Then each symbol is multiplied with one chip of the spreading code $c_{k}(l) \quad(1=\mathrm{m}=1,2, \ldots \mathrm{N})$ with length $\mathrm{N}_{\mathrm{k}}$ and chip duration $\mathrm{T}_{\mathrm{c}}$ and modulated to one of the $\mathrm{N}_{\mathrm{k}}$ subcarriers as shown in Fig.1,. The transmitted signal for the k-th user in $\mathrm{g}$-th group is given by

$$
S_{k, g}(n)=\sum_{m=1}^{N_{k}} \sqrt{2 P_{s}} b_{g, k}(n) c_{g, k}(m) \exp \left(j w_{m} t+\theta_{g, k, m}\right)
$$

where $P_{s}$ is the signal power of each subcarrier and is assumed to be the same for all users, $\mathrm{w}_{\mathrm{m}}=\mathrm{w}_{\mathrm{c}}+2 \pi \mathrm{m} / \mathrm{T}_{\mathrm{b}}$ is the $\mathrm{m}$-th subcarrier frequency, $\mathrm{w}_{\mathrm{c}}$ is the radio frequency and $\theta_{\mathrm{k}}$ is the random carrier phase uniformly distributed over $[0,2 \pi]$.

The transmitted signal is the sum of K users' spread data modulated on $\mathrm{N}_{\mathrm{c}}$ subcarriers given by

$$
s_{g}(t)=\sum_{k=1}^{K} \sum_{m=0}^{N_{c}-1} \sqrt{2 P_{s}} b_{g, k}(n) c_{g, k}(m) \exp \left(j w_{m} t+\theta_{g, k, m}\right)
$$

Each transmitted subcarrier signal passes through a frequency non-selective Rayleigh fading channel, and is 
also subjected to additive white Gaussian noise (AWGN) $n(t)$, with a double-sided power spectral density $\mathrm{N}_{\mathrm{o}} / 2$. For different users the statistics of all the Rayleigh fading channels are assumed to be identical. Furthermore, we assume that the channel fading is slow and remains unchanged over two consecutive symbols. The complex impulse response of the Rayleigh fading channel for the $\mathrm{m}$ th subcarrier can be written as

$$
h_{m}(t)=\beta_{m} e^{j \theta_{m}} \delta(t)
$$

where $\theta_{\mathrm{m}}$ is a random phase introduced by the channel, it is modeled as uniformly distributed over the interval of $[0$, $2 \pi]$ and is assumed to be i.i.d. for each subcarrier and each user, $\beta_{\mathrm{m}}$ is a Rayleigh random variable with power $E\left[\beta_{\mathrm{m}}^{2}\right]$ $=\sigma^{2}$ where E[.] is the expectation operator.

Given the above channel characteristics, the received signal for $\mathrm{k}$-th user can be written as

$$
\begin{aligned}
& r_{g}(n)=h_{g}(n) * s_{g}(n)+w(n) \\
= & \sum_{k=1}^{K} \sum_{m=0}^{N_{c}-1} \sqrt{2 P_{s}} \beta_{m} s_{k}\left(n-n_{k}\right) \exp \left(j w_{m} t+\phi_{m}\right)+w(n) \\
= & \sum_{k=1}^{K} \sum_{m=0}^{N_{c}-1} \sqrt{2 P_{s}} \beta_{m} b_{g, k}\left(n-n_{k}\right) c_{g, k}\left(n-n_{k}\right) \exp \left(j w_{m} t+\phi_{m}\right)+w(n)
\end{aligned}
$$

where $\mathrm{n}_{\mathrm{k}}$ is the propagation delay, uniformly distributed over $\left[0, \pm \mathrm{T}_{\mathrm{c}} / 2\right], \mathrm{w}(\mathrm{n})$ is the additive white Gaussian noise (AWGN) with zero mean and a double-sided power spectral density of $\mathrm{N}_{\mathrm{o}} / 2, \beta_{\mathrm{m}}$ and $\varphi_{\mathrm{m}}$ are the fading envelopes and random phase experienced by each user at subcarrier $\mathrm{m}$. $\beta_{\mathrm{m}}, \varphi_{\mathrm{m}}, \mathrm{n}_{\mathrm{k}}$ and $\mathrm{b}_{\mathrm{g}, \mathrm{k}}(\mathrm{t})$ are assumed to be independent and identically distributed (i.i.d.) for different $\mathrm{k}$.

In this system, each chip modulates a different carrier frequency. Therefore, the chip duration is same as the bit duration. In multicarrier modulation, each subcarrier experiences frequency non-selective fading. Each subcarrier is modulated with a narrowband signal whose bandwidth is smaller than the channel coherence. A combination of IDFT and a cyclic prefix at the transmitter with the DFT at the receiver converts the frequency selective channel to separate flat fading channels. Therefore, in frequency-selective fading channels, each subcarrier experiences flat fading that may be jointly correlated.

Channel estimation is used to compensate for the amplitude and phase distortions associated with the received signal. To estimate the multiplicative channel response, pilot symbols are inserted among the transmitted data symbols. The receiver estimates the channel state information based on the received, known pilot symbols. At the receiver, the guard intervals of the received signals are removed and the resultant symbol sequence is converted to a modulated signal of each subcarrier using FFT. For channel estimation, the channel impulse response at each subcarrier is found by averaging the impulse response measured for each of the dedicated pilot symbols. Using the obtained channel impulse response, the output of each subcarrier is equalized and then coherently combined over the $\mathrm{N}$ parallel subcarrier components i.e. despreading the signals. Finally, the regenerated symbol sequences are parallel to serial converted to recover the transmitted binary data.

Since the subchannel bandwidth is smaller, fading per subchannel is flat and low complex detection techniques can be used. We consider a correlation receiver, assuming perfect channel estimation for the desired user. In the receiver, the received signal is copied and fed to $N_{c}$ subcarriers. Each subcarrier is demodulated and despread by multiplying with one chip of the spreading code. The combined signal is then passed through a matched filter followed by a maximum likelihood detector.

Let $\mathrm{x}_{\mathrm{m}}$ be the summation of $\mathrm{K}$ users' transmitted $\mathrm{m}$-th chip during the i-th bit interval:

$$
x_{m}=\sum_{k=1}^{K} b_{i}^{k} c^{k}{ }_{i, m} \quad m=0,1, . . N-1
$$

where $c^{k}{ }_{i, m}$ is the k-th user's $\mathrm{m}$-th chip in the i-th bit interval. The sequence $\mathrm{x}_{\mathrm{m}}$ is parallel to serial converted and its IDFT is taken. The IDFT is

$$
w_{h}=\frac{1}{N} \sum_{m=1}^{N} x_{m} \exp (j 2 \pi m h / N), \mathrm{h}=0,1 \ldots \mathrm{N}-1
$$

The $\mathrm{P} / \mathrm{S}$ conversion and zero-th order interpolation gives the continuous-time signal

$$
w(t)=\sum_{h=0}^{N-1} w_{h} \cdot q\left(t-h T_{c}\right)
$$

where $\mathrm{q}(\mathrm{t})$ is the unit rectangular pulse over a chip interval. Let the normalized frequency offset $\varepsilon$ be $f_{o} / \Delta f$, where $f_{o}$ is a frequency offset and $\Delta \mathrm{f}=1 / \mathrm{NT}_{\mathrm{c}}$. At the receiver input, the noiseless component of the received signal during the bit interval, impaired by the frequency offset, is

$$
r(t)=\sum_{h=0}^{N-1} \sum_{m=0}^{N-1} x_{m} q\left(t-h T_{c}\right) \exp (j 2 \pi(m+\varepsilon) h / N)
$$




$$
r_{h}=\frac{1}{T_{c}} \int_{h T_{c}}^{(h+1) T_{c}} r(t) q\left(t-h T_{c}\right) d t \quad \quad \mathrm{~h}=0,1 \ldots . \mathrm{N}-1
$$

The frequency offset produces ICI. Thus the h-th DFT input is

$$
\begin{aligned}
& y_{h}=\frac{1}{N} \sum_{m=0}^{N-1} x_{m} \exp \left(j 2 \pi h \frac{m+\varepsilon}{N}\right) \\
& =\frac{1}{N} \sum_{m=0}^{N-1} \sum_{k=0}^{K} b_{i}^{k} c^{k}{ }_{i, m} \exp \left(j 2 \pi h \frac{m+\varepsilon}{N}\right)
\end{aligned}
$$

The $\mathrm{N}$ values of $\mathrm{y}_{\mathrm{h}}$ corrupted by AWGN noise and MAI are fed to the DFT. The DFT output is given by

$$
\begin{aligned}
z_{g} & =\sum_{h=1}^{N} y_{h} \exp (-j 2 \pi h g / N) \\
& =\sum_{h=0}^{N-1} \sum_{m=0}^{N-1} \sum_{k=0}^{K} b_{i}^{k} c^{k}{ }_{i, m} \frac{1}{N} \exp (j 2 \pi(m+\varepsilon-g) h / N) \\
& =\sum_{h=0}^{N-1} \sum_{m=0}^{N-1}\left[b_{i}^{d} c^{d}{ }_{i, m}+\sum_{k=0, k \neq d}^{K} b_{i}{ }^{k} c^{k}{ }_{i, m}\right] \frac{1}{N} \exp (j 2 \pi(m+\varepsilon-g) h / N)+\eta_{g}
\end{aligned}
$$

where $b^{d}{ }_{i}$ and $c^{d}{ }_{i, m}$ are the desired user's m-th chip in the i-th bit interval. $\eta_{\mathrm{g}}$ is the Gaussian noise variable.

$$
\begin{aligned}
& \sum_{g=0}^{N-1} c^{d}{ }_{i, g} z_{g}=b_{i}{ }^{d} \sum_{h=0}^{N-1} \frac{1}{N} \exp (j 2 \pi \varepsilon h / N) \\
& +b_{i}^{d} \sum_{g=0}^{N-1} \sum_{h=0}^{N-1} \sum_{\substack{m=0 \\
m \neq g}}^{N-1} c^{d}{ }_{i, g} c^{d}{ }_{i, m} \frac{1}{N} \exp (j 2 \pi(m+\varepsilon-g) h / N) \\
& +\sum_{g=0}^{N-1} \sum_{h=0}^{N-1} \sum_{\substack{k=0 \\
k \neq d}}^{K-1} b_{i}^{k} c^{d}{ }_{i, g} c^{k}{ }_{i, g} \frac{1}{N} \exp (j 2 \pi \varepsilon h / N) \\
& +\sum_{g=0}^{N-1} \sum_{h=0}^{N-1} \sum_{\substack{m=0 \\
m \neq g}}^{N-1} \sum_{\substack{k=0 \\
k \neq d}}^{K} b_{i}^{k} c^{d}{ }_{i, g} c^{k}{ }_{i, m} \frac{1}{N} \exp (j 2 \pi(m+\varepsilon-g) h / N)+\eta_{g}
\end{aligned}
$$

where the first, second, third and fourth terms at the right hand side represent the desired signal, self-interference, the MAI and the ICI respectively. Self interference is the interference from the desired user's own data in other chip intervals and is introduced due to non-zero frequency offset. The MAI is the interference from other users in the same
The DFT output is despread using the desired user's spreading sequence during the $\mathrm{i}$-th bit interval and we obtain chip interval. The ICI is the interference from other users in other chip intervals due to non-zero frequency offset. The estimated bit of the desired user can be obtained by using a QPSK demodulator followed by a hard limiter.

The variable of the MAI, ICI and noise for user $d$ during the i-th bit interval can be written as 


$$
\begin{gathered}
f_{i}^{d}=\sum_{g=0}^{N-1} c^{d}{ }_{i, g} z_{g}=\sum_{g=0}^{N-1} \sum_{h=0}^{N-1} \sum_{m=0}^{N-1} c^{d}{ }_{i, g} \sum_{\substack{k=0 \\
k \neq d}}^{K} b_{i}^{k} c^{k}{ }_{i, m} \frac{1}{N} \exp (j 2 \pi(m-g+\varepsilon) h / N)+c^{d}{ }_{i, g} \eta_{g} \\
=\sum_{g=0}^{N-1}\left\{\sum_{m=0}^{N-1} c^{d}{ }_{i, g} u_{m}+n_{g}\right\} S_{m} \\
=\sum_{m=0}^{N-1} \mu_{m} S_{m}=\mu_{0} S_{0}+\sum_{m=1}^{N-1} \mu_{m} S_{m}
\end{gathered}
$$

Where $\mu_{m}$ is the MAI in the $\mathrm{i}$-th bit and $\mathrm{m}$-th chip interval and $S_{m}$ is the attenuation factor due to the relative carrier frequency offset $\varepsilon$ given by

$$
\begin{gathered}
\mu_{m}=\sum_{\substack{k=0 \\
k \neq d}}^{K-1} b_{i}^{k} c^{k}{ }_{i, m} \\
f(x)=\frac{1}{2^{n K N}} \sum_{i_{0}=0}^{n K}, \ldots . . \sum_{i_{N-1}=0}^{n K}\left(\begin{array}{l}
n K \\
i_{0}
\end{array}\right), \ldots . .\left(\begin{array}{l}
n K \\
i_{N-1}
\end{array}\right) \\
\sigma_{I}^{2}=\sigma^{2} \sum_{k=0}^{N-1} S_{k}^{2}+\frac{K}{N} A^{2} \sum_{k=0}^{N-1} \alpha^{2} S_{k}^{2}
\end{gathered}
$$$$
S_{m}=\frac{1}{N} \sum_{h=0}^{N-1} \exp \{j 2 \pi(m+\varepsilon) h / N\}
$$$$
=\frac{\sin (\pi(m+\varepsilon))}{N \sin \left(\frac{\pi}{N}(m+\varepsilon)\right)} \exp \left(j\left\{\pi\left(1-\frac{1}{N}\right)(m+\varepsilon)\right\}\right)
$$

The conditional pdf of the MAI, ICI and noise in frequency selective fading channels given the fading vector, $\vec{\alpha}=\left\{\alpha_{0}, \ldots \ldots . . \alpha_{N-1}\right\}$, where $\alpha_{\mathrm{i}}$ is the fading parameter in the $\mathrm{i}$-th subcarrier is obtained as weighted sum of $\mathrm{nKN}$ Gaussian pdfs

$$
f(x)=\frac{1}{2^{n K N}} \sum_{i_{0}=0}^{n K}, \ldots . . \sum_{i_{N-1}=0}^{n K}\left(\begin{array}{l}
n K \\
i_{0}
\end{array}\right), \ldots . .\left(\begin{array}{l}
n K \\
i_{N-1}
\end{array}\right) \frac{1}{\sqrt{2 \pi \sigma^{2} \sum_{k=0}^{N-1} S_{k}^{2}}} \exp \left\{-\frac{\left\{x-A\left[K \sum_{k=0}^{N-1} \alpha_{k} S_{k}-2\left(\sum_{k=0}^{N-1} i_{k} \alpha_{k} S_{k} / n\right]\right\}^{2}\right)}{2 \sigma^{2} \sum_{k=0}^{N-1} S_{k}^{2}}\right\}
$$

The variance is found to be without fading can be obtained by replacing $\mathrm{S}_{0}=1, \mathrm{~S}_{\mathrm{k}}=0$ and $\alpha_{\mathrm{k}}=1$ for all $\mathrm{k}$ as

The pdf and variance without frequency offset for and

$$
\begin{aligned}
& \left.f(x)=\frac{1}{2^{N K}} \sum_{l=0}^{N K}\left(\begin{array}{l}
N K \\
l
\end{array}\right) \frac{1}{\sqrt{2 \pi \sigma^{2}}} \exp \left\{-[x-A(K-2 l / N)]^{2} / 2 \sigma^{2}\right)\right\} \\
& \sigma_{I}^{2}=\sigma^{2}+\frac{K A^{2}}{N} \quad=\int_{0}^{\infty} Q\left(\sqrt{\sum_{i=0}^{N-1} \frac{S_{0}^{2} \gamma_{i}\left(E_{b} / N_{o}\right)}{\sum_{k=0}^{N-1} S_{k}^{2}+\frac{K}{n}\left(E_{b} / N_{o}\right) \sum_{j=0}^{N-1} \gamma_{j} S_{j}^{2}}}\right) f_{\gamma}(\gamma) d \gamma
\end{aligned}
$$

Equations 21 and 22 are derived assuming Gaussian approximations of MAI and ICI.

Then the Gaussian approximation of BER is obtained as

$$
P_{e}=\int_{0}^{\infty} Q\left(\sqrt{\sum_{i=0}^{N-1} \frac{S_{0}^{2} \gamma_{i} A^{2} / n}{\sigma^{2} \sum_{k=0}^{N-1} S_{k}^{2}+\frac{K}{n} A^{2} \sum_{j=0}^{N-1} \gamma_{j} S_{j}^{2}}}\right) f_{\gamma}(\gamma) d \gamma
$$

For flat fading, $\gamma_{i}=\gamma=\alpha^{2}$ for all $\mathrm{i}$ and the equation for BER reduces to

$$
P_{e}=\int_{0}^{\infty} Q\left(\sqrt{\sum_{i=0}^{N-1} \frac{S_{0}^{2} \gamma\left(E_{b} / N_{o}\right)}{\left(1+\frac{K}{n} \gamma\left(E_{b} / N_{o}\right)\left(\sum_{j=0}^{N-1} S_{j}^{2}\right)\right.}}\right) e^{-\gamma} d \gamma
$$


For AWGN channels, $\gamma_{\mathrm{i}}=1$ and equation (20) further reduces to

$$
P_{e}=Q\left(\sqrt{\frac{S_{0}^{2}\left(2 E_{b} / N_{o}\right)}{\left(1+\frac{K}{n} \frac{2 E_{b}}{N_{o}}\right)\left(\sum_{j=0}^{N-1} S_{j}^{2}\right)}}\right)
$$

The MAI term can be removed for orthogonal spreading systems. However, the MAI is not completely removed in frequency selective fading channels even when orthogonal spreading sequences are employed. The fading in each carrier is not identical and may be correlated. At the receiver, the different amplitude in each chip may destroy the orthogonality of the sequences.

\section{Simulation Results}

Simulations are also conducted to evaluate error performance of the proposed multiple access scheme. Monte-Carlo simulation method is applied to estimate the bit error rate of the proposed scheme. The BER performance is analyzed and simulated for three different cases: low data rate $(8 \mathrm{kbps})$ users having a high spreading factor of 32, high (10 Mbps) data rate users having a lower spreading factor of 4 and the average BER performance, where users at different data rates use different spreading factors. It is found that the performance is better when transmission is at low data rates than at high data rates since at low rates, a higher spreading factor is used which reduces the MAI.

The simulation parameters are summarized in Table.1. In each frame, time slots are assigned to users independently in accordance with their data rates. The data rates vary from $8 \mathrm{kbps}$ to $10 \mathrm{Mbps}$. The modulation used is QPSK and spreading factor is chosen to be $4,8,16$ or 32 depending on the data rates. Walsh codes are used since they are orthogonal. The RF bandwidth is assumed to be $20 \mathrm{MHz}$ and the total number of subcarriers is 1024 with a subcarrier spacing of $19.53 \mathrm{KHz}$. The ITU-R Vehicular Channel A model is used for simulation.

Table.1. Simulation parameters

\begin{tabular}{ll}
\hline Parameters & Values \\
\hline Bandwidth & $80 \mathrm{MHz}$ \\
Data Rate & $8 \mathrm{Kbps}$ to $10 \mathrm{Mbps}$ \\
Spreading Codes & Walsh Codes \\
Spreading Factor & $4,8,16,32$ \\
Modulation & QPSK \\
Number of subcarriers & 1024 \\
Subcarrier spacing & $78.125 \mathrm{KHz}$ \\
\hline
\end{tabular}

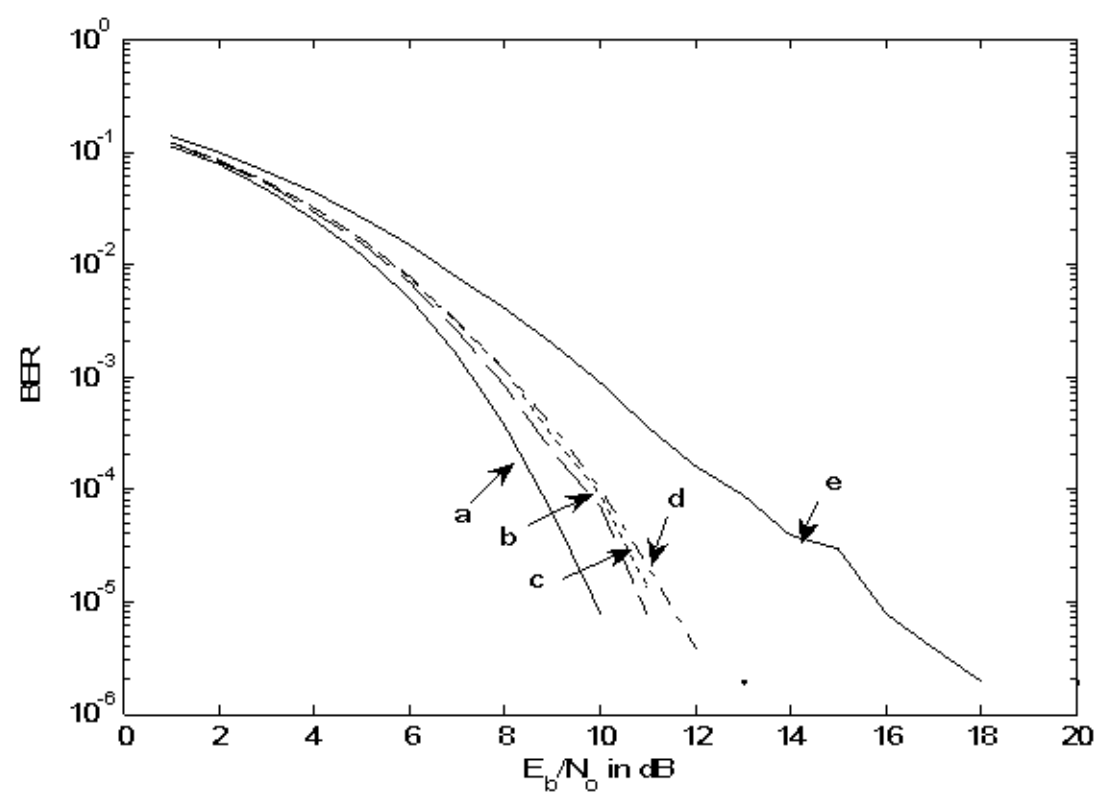

Fig.4. BER performance of the TD-MC-CDMA multiple access system in downlink for a data rate of 8 kbps (a) Simulation result for BER of 1 to 16 users in AWGN channel (b) Simulation result for BER of 1 user in fading channel (c) Simulation result for BER of 4 users in fading channel (d) Simulation result for BER of 16 users in fading channel and (e) Analytical result for BER of 16 users in fading channel.

Fig.4 shows the BER performance of the TD-MCCDMA scheme in synchronous downlink in AWGN and fading channels for a data rate of $8 \mathrm{kbps}$ and a spreading factor of 32. It is found that the BER performance is same for any number of users in AWGN channel since the codes are orthogonal and there is no interference. However, in a multipath fading channel, the performance degrades as the number of users increases due to multiple access interference. The required $\mathrm{E}_{\mathrm{b}} / \mathrm{N}_{\mathrm{o}}$ to get a BER of $10^{-4}$ is 13 $\mathrm{dB}$ for single user, $13.5 \mathrm{~dB}$ for 4 users and it is $14 \mathrm{~dB}$ for 16 
users. Walsh codes are used which are orthogonal, but the MAI is not zero since each chip of the PN sequence experiences independent fading, which tends to destroy the orthogonality between spreading sequences. This increases the MAI and degrades the BER performance. However, the degradation is very small as the number of active users increases. The simulation results are in good agreement with the predictions of analytical results.

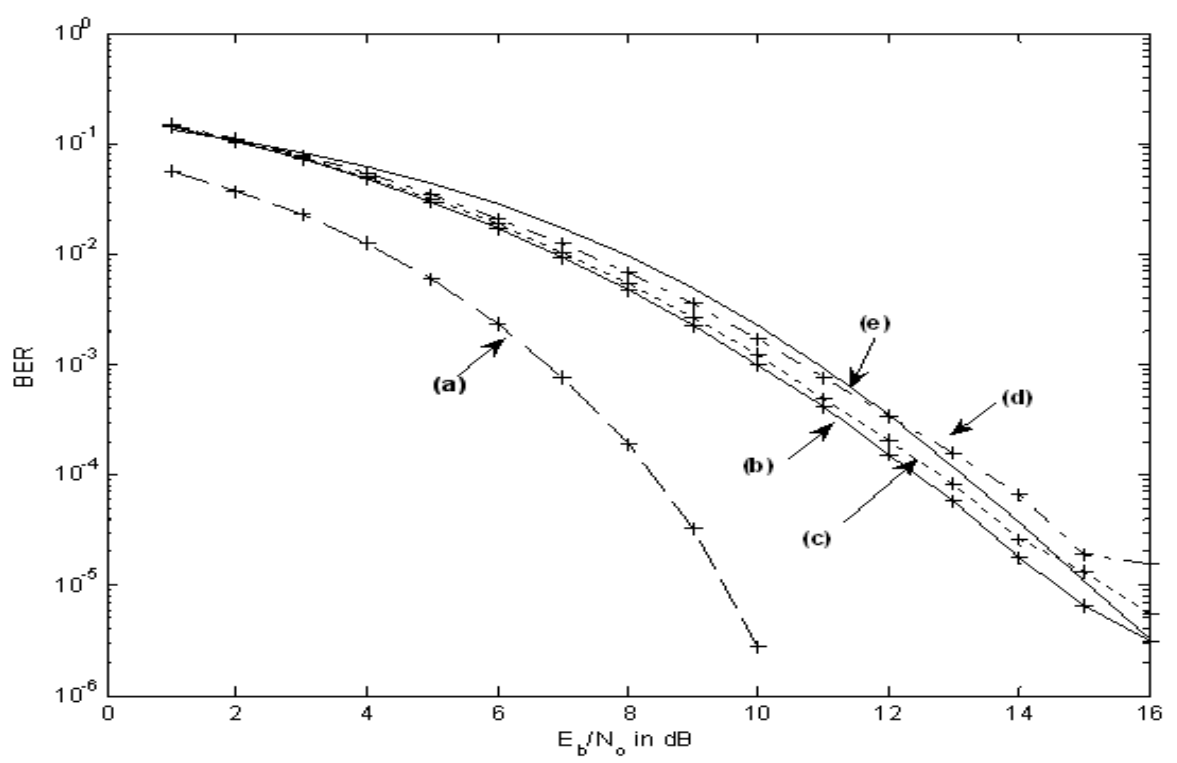

Fig.5. BER performance of the TD-MC-CDMA multiple access system in downlink for a data rate of 10 Mbps (a) Simulation result for BER of 1 to 4 users in AWGN channel (b) Analytical result for BER of one user in fading channel (c) Simulation result for BER of 1 user in fading channel (d) Simulation result for BER of 2 users in fading channel and (e) Simulation result for BER of 4 users in fading channel.

Fig.5 shows the BER performance of the TD-MCCDMA scheme in synchronous downlink in AWGN and fading channels for a data rate of $10 \mathrm{Mbps}$ and a spreading factor of 4. It is found that the BER performance is same for any number of users in AWGN channel since the codes are orthogonal and there is no interference. In a multipath fading channel, the required $\mathrm{E}_{\mathrm{b}} / \mathrm{N}_{\mathrm{o}}$ to get a BER of $10^{-3}$ is
$17 \mathrm{~dB}$ for single user, $20 \mathrm{~dB}$ for 2 users and it is about 25 $\mathrm{dB}$ for 4 users. Since the spreading factor is less, the MAI is more in this case. The degradation in BER performance is also more as the number of users increases due to multiple access interference. The simulation results are in good agreement with the predictions of analytical results.

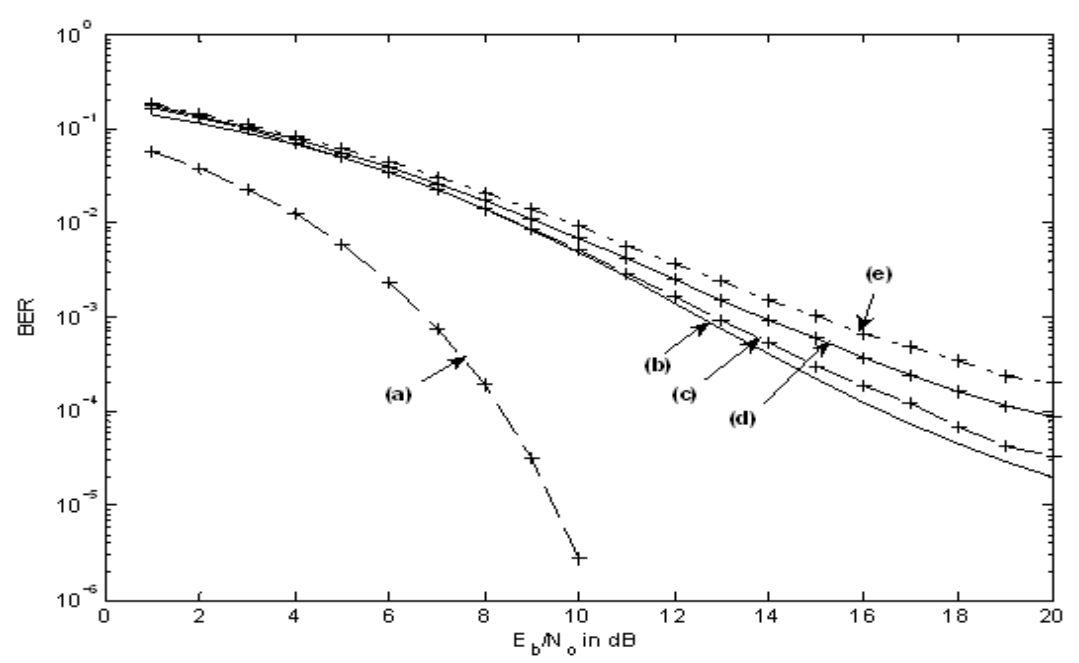

Fig.6. Average BER performance of the TD-MC-CDMA multiple access system in downlink (a) Simulation result for BER of 1 to 16 users in AWGN channel (b) Analytical result for BER of 4 users in fading channel (c) Simulation result for BER of 4 users in fading channel (d) Simulation result for BER of 8 users in fading channel and (e) Simulation result for BER of 16 users in fading channel.

Fig. 6 shows the average BER performance of the TDMC-CDMA scheme in synchronous downlink in AWGN and fading channels for a number of users having data rates from $8 \mathrm{kbps}$ to $10 \mathrm{Mbps}$. It is found that the BER performance degrades as the number of users increases due to multiple access interference. The required $\mathrm{E}_{\mathrm{b}} / \mathrm{N}_{\mathrm{o}}$ to get a BER of $10^{-4}$ is $13 \mathrm{~dB}$ for 4 users, $14 \mathrm{~dB}$ for 8 users and it is $16 \mathrm{~dB}$ for 16 users. However, the degradation is not very 
large as the number of active users increases. The simulation results are in good agreement with the predictions of analytical results.

\section{Conclusions}

TD-MC-CDMA scheme can be used for $4 \mathrm{G}$ cellular systems with spreading in frequency domain for downlink and spreading in time domain for uplink. 3GPP LTE and WiMAX Mobile use OFDMA for multiple access. The proposed scheme can give better diversity and flexibility for transmission of different classes of data e.g. audio, video, internet, ISDN, multimedia etc. having multiple data rates. The parameters e.g. spreading factor, number of time slots, number of subcarriers etc. are chosen according to the required data rate, the available bandwidth, the number of subscribers etc. The performances of proposed system for uplink and downlink channels have been studied. Expressions for multiple access interference and bit error rate are derived. The simulation results are in good agreement with the predictions of theoretical/ analytical results. Furthermore, simulations imply that MAI, that determines the error rate, can be approximated as Gaussian for many practical cases.

\section{References}

[1] H.Sari and G. Karam, "Orthogonal Frequency Division Multiple Access and its Applications to CATV Networks," Eur. Trans. Comm., vol.45, pp 507-516, Nov.-Dec., 1998.

[2] S. H. Ali, K. Lee and V. C. M. Leung, "Dynamic Resource Allocation in OFDMA Wireless Metropolitan Area Networks", IEEE Wireless Communication, Feb, 2007.

[3] K. H. Teo, Z. Tao, and J. Zhang "The Mobile Broadband WiMAX Standard," IEEE Signal Processing Magazine, September, 2007.

[4] The WiMax Forum (www.wimaxforum.org)

[5] K. Fazel and S. Kaiser, Multi-Carrier and Spread Spectrum Systems, John Wiley and sons Ltd., 2003.

[6] L. Hanzo, B. J. Choi, Thomas Keller, and M. Mnster, OFDM and MC-CDMA for Broadband Multi-User Communications, WLANs and Broadcasting, John Wiley and Sons, 2003.

[7] L. Hanzo, L. L. Yang, E. L. Kuan, K. Yen, Single and MultiCarrier DS-CDMA: Multiuser Detection, Space Time Spreading, Synchronization, Networking and Standards, IEEE Press, 2005.
[8] S. Hara and R. Prasad, "Overview of multicarrier CDMA," IEEE Communication magazine, vol.35, pp.126-133, Dec., 1997.

[9] S. Hara and R. Prasad, "Design and Performance of Multicarrier CDMA System in Frequency-Selective Rayleigh Fading Channels," IEEE Transactions on Vehicular Technology, vol. 48, No. 5, pp.1584-1595, Sept. 1999.

[10] Tobias Weber, Johannes Schlee, Stefan Bahrenburg, Paul Walter Baier, Jürgen Mayer and Christoph Euscher, "A Hardware Demonstrator for TD-CDMA," IEEE Transactions On Vehicular Technology, Vol. 51, No. 5, September 2002.

[11] M. Haardt, A. Klein, R. Koehn, S. Oestreich, M. Purat, V. Sommer, T. Ulrich, "The TD-CDMA based UTRA TDD mode, " IEEE Journal on Selected Areas in Communications, vol. 18 August 2000.

[12] 3GPP Specifications-Releases available at http://www.3gpp.org

[13] M. Chang and Y. T. Su, "Performance Analysis of Equalized OFDM Systems in Rayleigh Fading", IEEE Transactions on Wireless Communications, vol. 1, No.4, Oct. 2002.

[14] A. Chouly, A. Brajal, and S. Jourdan, "Orthogonal multicarrier techniques applied to direct sequence spread spectrum CDMA systems," Proc. IEEE GLOBECOM'93, pp.1723-1728, Nov., 1993.

[15] K. Fazel, "Performance of CDMA/OFDM for mobile communication system," Proc. IEEE ICUPC'93, pp. 975979, Oct., 1993.

[16] Z. Hou and V. K. Dubey, "Bit Error Rate Probability of MCCDMA System over Rayleigh Fading Channels", ISSSTA2004, Sydney, Australia, 30 Aug.-2 Sep. 2004.

[17] H. Ishikawa, M. Furudate, T. Ohseki and T. Suzuki, "Performance Analysis of Adaptive Downlink Modulation Using OFDM and MC-CDMA for Future Mobile Communication System", ISSSTA2004, Sydney, Australia, 30 Aug.-2 Sep. 2004.

[18] S. Kondo and L.B. Milstein, "Performance of multicarrier DS-CDMA systems," IEEE transactions on Communications, vol. 44, no.2, pp.238-246, Feb., 1996.

[19] E. A. Sourour and M. Nakagawa, "Performance of orthogonal multicarrier CDMA in a multipath fading channel," IEEE Transactions on Communications, vol. 44, no. 3, pp.356-367, March, 1996.

[20] S. Suwa, H. Atarashi, and M. Sawahashi, "Performance Comparison Between MC/DS-CDMA and MC-CDMA for Reverse Link Broadband Packet Wireless Access," IEEE Vehicular Technology Conference, 2002 Proceedings vol.4 pp. 2076-2080, Sept. 2002. 